\title{
Neuropsychological Correlates and Frequency of Tinnitus in Patients Injured in Motor Vehicle Accidents
}

\author{
Zack Z. Cernovsky, Stephan C. Mann, and Varadaraj R. Velamoor
}

\section{ABSTRACT}

Background: There is a lack of data on the frequency and neuropsychological correlates of tinnitus in distinct clinical populations such as persons injured in high impact motor vehicle accidents (MVAs).

Method: Tinnitus severity and frequency were analyzed statistically in deidentified data of 106 post-MVA patients (mean age=39.5, $\mathrm{SD}=13.1 ; 31$ males and 75 females). Correlations of tinnitus to the patients' scores on the Rivermead Post-Concussion Symptoms Questionnaire, Subjective Neuropsychological Symptoms Scale (SNPSS), Brief Pain Inventory, Insomnia Severity Index, PTSD measure (PCL-5), and to ratings of depression, anger, and anxiety on the Whiplash Disability Questionnaire were evaluated. The patients were interviewed an average of 53.6weeks (SD=39.9) after their MVA; all continued to suffer from post-MVA symptoms requiring therapy.

Results: Tinnitus was reported by 70 of the 106 post-MVA patients $(66 \%)$. Slightly less than a half of the $\mathbf{7 0}$ rated their tinnitus as "frequent" or "persistent" and slightly more than a half as "occasional."

With respect to Rivermead and SNPSS items, the severity and frequency of tinnitus correlated significantly (at $p<.01,2$-tailed) with oversensitivity to light, restlessness, word finding difficulty, hand tremor, stutter, and instances of reduced muscular control over hand or arm (rs ranging from .28 to .37). Tinnitus severity correlated also with tingling in the limbs $(r=.31)$, and with impaired balance $(r=.28)$. Tinnitus frequency correlated also with difficulty articulating words $(r=.28)$. Tinnitus also significantly correlated with ratings of pain and of insomnia (rs ranging from .26 to .32), but not with variables such as PTSD, depression, or anxiety.

Discussion and Conclusions: Tinnitus is correlated with some aspects of whiplash trauma and the post-concussion syndrome. However, the size of these relationships is rather weak.

Keywords: tinnitus, post-concussion syndrome, whiplash syndrome, neuropsychology.

\section{INTRODUCTION}

Tinnitus can be defined as the perception of sound for which there is no detectable acoustic source external to the head. Tinnitus can be experienced as a ringing, buzzing, humming, hissing, or various other types of sound. Patients experience tinnitus as present constantly, or frequently, or only occasionally, or rarely. Some patients describe the sound as high-pitched.

Studies estimate that the prevalence of tinnitus in the normal population of adults may be within the range of $10 \%$ to $15 \%$ [1]. With respect to age groups, the peak in tinnitus prevalence seems to be among sexagenarians and septuagenarians, i.e., between the age of 60 and 79 [2].

Tinnitus caused by trauma such as head and neck injuries in MVAs is referred to as posttraumatic tinnitus. Head or auditory processing.
Submitted : March 15, 2021

Published : April 05, 2021

ISSN: 2593-8339

DOI: $10.24018 /$ ejmed.2021.3.2.771

\section{Z. Z. Cernovsky*}

Dept. of Psychiatry, Western University, London, Ontario, Canada.

(e-mail: zcernovs@uwo.ca)

S. C. Mann*

Central Montgomery Behavioral Heath, Norristown, PA, USA.

(e-mail: smann1234@aol.com)

V. R. Velamoor

Dept. of Psychiatry, Laurentian and Lakehead Universities, and Western University, Ontario, Canada.

(e-mail: velamoorraj@ gmail.com)

*Corresponding Authors

cervical trauma can affect blood flow, auditory nerves, the inner ear, and the auditory pathway, and also the central

Head trauma may cause labyrinthine concussion with symptoms such as hearing loss, tinnitus, and dizziness. Research on labyrinthine concussions by Choi's team [3] indicated that patients who concurrently experience dizziness might have a less favourable outcome in their recovery from hearing loss. These investigators [3] also noted that the hearing loss of the post-concussive patients was more severe for high frequency than low frequency sounds.

The present study evaluates the relationship of tinnitus and its characteristics to various subjective neuropsychological variables relevant to the assessments of post-concussion and whiplash syndromes in post-MVA patients. In addition, it examines the relationships of tinnitus to pain, insomnia, PTSD, depression, and anxiety caused by the MVA. 


\section{METHOD}

The measures of tinnitus were available as a part of deidentified archival data on 106 survivors of motor vehicle accidents (MVAs). The group consisted of 31 males and 75 females, age 19 to 60 years (mean age $=39.5, \mathrm{SD}=13.1$ ). Their average scores were $20.3(\mathrm{SD}=10.2)$ on the Subjective Neuropsychological Symptoms Scale (SNPSS) [4], 6.4 $(\mathrm{SD}=1.5)$ on the "average pain" item of the Brief Pain Inventory [5], and 23.8 ( $\mathrm{SD}=4.3)$ on Morin's Insomnia Severity Index [6]. Scores on the Rivermead Post-Concussion Symptoms Questionnaire [7], [8] were available for 53 of our 106 patients. The Rivermead scores classified all 53 as experiencing some degree of the post-concussion syndrome (scores ranging from 24 to 60 on the Rivermead PostConcussion Symptoms Questionnaire [7], [8], with mean=46.4, SD=9.2).

All 106 patients provided ratings on Items 10 to 12 of the Whiplash Disability Questionnaire, [9] i.e., ratings of depression, anger, and of anxiety via scale from 0 ("not at all") to 10 ("always"). Scores on the PCL-5 measure of PTSD [10] were available for 32 of the 106 patients.

The time elapsed since the patient's MVA ranged from 9 to 194 weeks, with a mean of 53.6 weeks $(S D=39.9)$, i.e., on the average about a year after their MVA. All patients continued to experience active post-accident symptoms that required therapy, including post-accident pain, insomnia, PTSD, depression, generalized anxiety, and driving anxiety.

Only patients who did not experience tinnitus as a preexisting condition prior to the study were included. In addition, patients who had started to experience tinnitus in the aftermath of the accident, but had been free of tinnitus over the past one month prior to the study were excluded.

Among the 106 post-MVA patients, 73 had no previous MVA, 29 had one MVA, and 4 patients had more than one prior MVA. In their recent accident, 76 were the drivers, 18 were passengers, one drove a motorcycle, and 11 were pedestrians. In 38 cases, the collision involved the side of their vehicle, 31 experienced collision to the rear of their vehicle, 24 to the front, and other types of collisions occurred in 13 cases.

Information regarding loss of consciousness in the MVA was available for 104 of the 106 patients: 39 assumed they did not lose consciousness, although in similar situations brief episodes of unconscious can go unnoticed. Of the remaining patients, 42 were uncertain, 22 reported a brief loss of consciousness, and one lost consciousness for longer than 5 minutes. Their scores on the Immediate Concussion Symptoms (ICS) scale [11] ranged from 0 to 8 points (mean=4.6, $\mathrm{SD}=1.9)$.

\section{RESULTS}

\section{A. Severity of Tinnitus}

In this sample, $70(66.0 \%$, i.e., about $2 / 3)$ of the 106 postMVA patients reported tinnitus. Twenty-five (23.6\%) described the tinnitus as a mild problem, $4(3.8 \%)$ as mild to moderate, $26(24.5 \%)$ as moderate, and $15(14.2 \%)$ as severe.

\section{B. Tinnitus Frequency}

The patients rated the frequency of their tinnitus on the following scale: absent, rare, occasional, frequent, persistent. Thirty-six patients $(34.0 \%)$ reported no tinnitus, one patient $(0.9 \%)$ rated the tinnitus as rare to occasional, 39 patients $(36.8 \%)$ as occasional, one patient $(0.9 \%)$ rated the tinnitus as occasional to frequent, 17 patients $(16.0 \%)$ as frequent, and 12 patients $(11.3 \%)$ as persistent. Thus, about a third of postMVA patients reported no tinnitus, slightly less than a third reported a frequent or persistent tinnitus, and slightly more than a third experienced tinnitus only occasionally.

The ratings of tinnitus frequency were highly correlated with tinnitus severity $(\mathrm{r}=.92, \mathrm{p}<.001)$.

\section{Nature of the Sound of Tinnitus}

The patients were asked if their tinnitus seemed to be a ringing sound, or a buzzing one, or hissing, humming, highpitched, or other. The majority $43(40.6 \%)$ of patients rated the sound as a ringing one. The next most frequent descriptors were high-pitched (27 patients, i.e., $25.5 \%)$, buzzing, (25, i.e., $23.6 \%$ ), humming (10 or $9.4 \%$ ), and hissing ( 7 or $6.6 \%$ ). Five patients rated the sound as "other."

Nearly one-third of the 106 patients $(30.2 \%)$ chose only one of the 5 above adjectives to describe their tinnitus and roughly another third $(34.9 \%)$ used 2 or more of the 5 . The most frequent combination of 2 of the 5 adjectives was the "ringing + high pitched" (reported by $17.0 \%$ of the 106 patients).

As mentioned, 5 patients described the sound as "other" than the adjectives about which we specifically inquired. Each of these 5 patients described the sound in his or her own way via the following words: beeping, muffled, popping popcorn, screeching or crickets, and static.

\section{Neuropsychological Correlates of Tinnitus: Rivermead Items}

Scores on the Rivermead Post-Concussion Symptoms Questionnaire were available for only 53 of our 106 patients. With samples of this size or larger, weak correlations of potentially spurious nature may become statistically significant at $\mathrm{p}<.05$. For this reason, we interpreted here only correlations significant at $\mathrm{p}<.01,2$-tailed.

The Rivermead assesses the following 16 variables: headaches, dizziness, nausea, oversensitivity to noise, disturbed sleep, fatigue, irritability, depression, impatience, impaired memory, impaired concentration, slow speed of thinking, blurred vision, oversensitivity to light, double vision, and restlessness. Patients are asked if any of these symptoms were present within the last one month. Each of these variables is rated according to the extent of problem it caused to the patient: $0=$ not experienced at all, $1=$ no more of a problem (i.e., not experienced anymore within the last one month), 2=mild problem, 3=moderate problem, 4=severe problem. The total Rivermead score is calculated by adding all 16 variables.

The severity of tinnitus correlated significantly with the Rivermead total score ( $\mathrm{r}=.38)$. With respect to its 16 individual Rivermead items, the severity of tinnitus correlated significantly only with oversensitivity to light $(\mathrm{r}=.38)$ and with restlessness $(\mathrm{r}=.44)$. 
The frequency of tinnitus correlated only weakly with the total Rivermead score $(\mathrm{r}=.30)$ and this correlation failed our criterion of significance (the $\mathrm{p}$ was .027). With respect to individual Rivermead items, the tinnitus frequency correlated significantly with oversensitivity to light $(\mathrm{r}=.36)$ and restlessness $(r=.36)$.

It is noteworthy that our data identified no significant correlations between tinnitus and dizziness. Dizziness was highlighted as a variable of potential prognostic significance in the study by Choi et al. [3]. However, the lack of significant correlations of tinnitus to dizziness in our study could be due to our cross-sectional rather than longitudinal design: no information on follows up outcomes was available in the deidentified archival data of our patients.

\section{E. Neuropsychological Correlates of Tinnitus: SNPSS Items}

Scores on the Subjective Neuropsychological Symptoms Scale (SNPSS) were available for all patients in our sample. The SNPSS assesses the following 13 variables: impaired balance, hand tremor, instances of reduced muscular control over leg muscles, instances of reduced muscular control over arm or hand muscles, tingling in some of the limbs, numbness in some of the limbs, reduced feeling in some of the limbs, diminished bladder control, diminished bowel control, stutter, the syndrome of word finding difficulty, difficulties articulating words, and tinnitus. Each of these variables is rated according to the extent of problem it causes to the patient: $0=$ not experienced at all, $1=$ no more of a problem (i.e., not experienced anymore within the last one month), $2=$ mild problem, $3=$ moderate problem, $4=$ severe problem. The total score is normally calculated by adding all 13 variables. However, in the present study, we calculated the total score on the SNPSS without including in it the score on its tinnitus item, to avoid undue inflation of the correlation coefficient. This revised SNPSS score correlated significantly with tinnitus severity $(\mathrm{r}=.43)$ and with tinnitus frequency $(\mathrm{r}=.41)$.

With respect to individual items of the SNPSS, the severity of tinnitus correlated significantly with the syndrome of word finding difficulty $(\mathrm{r}=.37)$, hand tremor $(\mathrm{r}=.36)$, stutter $(\mathrm{r}=.32)$, instances of reduced muscular control over hand or arm $(\mathrm{r}=.32)$, tingling in the limbs $(\mathrm{r}=.31)$, and impaired balance $(\mathrm{r}=.28)$.

The frequency of tinnitus correlated significantly with stutter $(\mathrm{r}=.36)$, the syndrome of word finding difficulty $(\mathrm{r}=.35)$, hand tremor $\mathrm{r}=.31)$, instances of reduced muscular control over hand or arm ( $\mathrm{r}=.28)$, and difficulty articulating words $(\mathrm{r}=.28)$.

\section{F. High Pitch Tinnitus versus Neuropsychological Variables}

As mentioned previously, Choi et al. [3] found that hearing loss after labyrinthine concussion was more severe for high than for low frequency sounds. In our study, of the 70 patients who reported having tinnitus, 27 described it as high pitched and 43 as not high pitched. It is of interest to examine whether the pitch of the tinnitus is related in any manner to the neuropsychological variables in our cross-sectional data. Our dichotomous variable (high pitched versus not high pitched tinnitus) did not correlate significantly with any of the 16 Rivermead or 12 SNPSS variables (i.e., those other than tinnitus). Similarly, no significant correlation of the dichotomous variable (high pitched versus not high pitched tinnitus) was found to the total score on the Rivermead scale and the total score on the SNPSS (as mentioned, the total score on the SNPSS was calculated without including the score on its tinnitus item). However, the lack of significant correlations of the high pitched tinnitus to neuropsychological variables in our study could be due to our cross-sectional rather than longitudinal design: as already mentioned, no information on follow up outcomes was available in the archival data of our patients.

\section{G. Correlations of Tinnitus to Measures of Pain, Insomnia, PTSD, Depression, and Anxiety}

The severity of tinnitus correlated significantly with the ratings of average pain $(\mathrm{r}=.28)$, worst pain $(\mathrm{r}=.27)$, and the least pain $(\mathrm{r}=.26)$ on the Brief Pain Inventory.

The frequency of tinnitus correlated significantly with the ratings of average pain $(r=.29)$ and of the worst pain $(r=.27)$.

Total scores on the Insomnia Severity Index (ISI) were significantly correlated both with tinnitus severity $(r=.32)$ and tinnitus frequency $(\mathrm{r}=.28)$.

Neither tinnitus severity nor frequency correlated significantly with ratings of depression, generalized anxiety, and anger (items 10 to 12 of the Whiplash Disability Questionnaires).

No significant correlation was found of tinnitus severity or frequency to the PCL-5 measure of PTSD, however, the PCL5 measures were available for 32 patients only, i.e., a too small sample to allow for generalizations of these results.

\section{H. Correlations of Tinnitus to Age, Gender, Number of Weeks since the MVA, and Number of Prior MVAs}

No significant correlations were found of tinnitus severity or frequency to any of these variables.

\section{DISCUSSION}

Approximately two-thirds $(66 \%)$ of our post-MVA patients reported tinnitus. Only 15 of the 106 patients (14.2\%) considered their tinnitus a severe problem. Twenty-nine patients $(27.3 \%)$ rated their tinnitus as frequent or persistent. Choi's team [3] studied 40 patients, 35 of whom sustained head injury in traffic accidents, 3 in assaults, and two in falls. There were $22(55 \%)$ bilaterally affected and $18(45 \%)$ unilaterally affected. Unfortunately, our own study has not differentiated between bilateral and unilateral tinnitus, in order to compare the frequencies.

Correlational relationships of tinnitus found to other neuropsychological variables in the present study are mostly weak. Only a few could be considered as of moderate strength. These correlates of tinnitus include a varied mixture of subjective neuropsychological variables such as oversensitivity to bright lights, the syndrome of word finding difficulty, hand tremor, instances of reduced muscular control over hand or arm, tingling in the limbs, impaired balance, stutter, difficulties articulating words, and restlessness. This suggests that tinnitus may be related to some aspects of whiplash trauma and of the post-concussion syndrome, however, the size of these relationships to individual 
neuropsychological symptoms is weak.

Tinnitus was not significantly correlated with variables such as PTSD, depression, or anxiety, but correlated significantly with ratings of pain and of insomnia. It is possible that the persistent pain and insomnia interfere with medical recovery from the post-accident tinnitus.

\section{CONCLUSIONS}

Although our 106 post-MVA patients were assessed, on the average, about a year after their car accident, about two thirds reported that they still experience tinnitus. Tinnitus was rated as persistent or frequent by $27.3 \%$ of the 106 patients. Tinnitus was significantly correlated with several neuropsychological variables in the post-concussion and whiplash spectrum, however, the size of these correlations was only weak to moderate.

Tinnitus correlated positively with ratings of pain and insomnia: it is possible that persistent pain and insomnia obstruct the recovery from tinnitus.

\section{ACKNOWLEDGMENT}

The authors thank to Abe Cernovsky, BA, for his editorial assistance in this research project.

\section{REFERENCES}

[1] Henry JA, Dennis KC, Schechter MA. General Review of Tinnitus: Prevalence, Mechanisms, Effects, and Management. Journal of Speech, Language, and Hearing Research. 2005;48:1204-1235.

[2] Hoffman HJ, \& Reed GW. Epidemiology of tinnitus. In J. B. Snow, Jr. (Ed.), Tinnitus: Theory and management. Lewiston, NY: BC Decker, 2004, pp. 16-41.

[3] Choi MS, Shin S-O, Yeon JY, Choi YS, Kim J, and Park SK. Clinical Characteristics of Labyrinthine Concussion. Korean Journal of Audiology. 2013;17:13-17.

[4] Cernovsky ZZ, Litman LC, Mann SC, Oyewumi LK, Bureau Y, Mendonça JD, Diamond DM, and Raheb H. Validation of the Subjective Neuropsychological Symptoms Scale (SNPSS) in Injured Motorists. Archives of Psychiatry and Behavioral Sciences. 2021;4(1):6-13.

[5] Cleeland CS. The Brief Pain Inventory - User Guide. Houston, TX: The University of Texas - M. D. Anderson Cancer Center, 2009.

[6] Morin CM, Belleville G, Bélanger L, and Ivers H. The insomnia severity index: psychometric indicators to detect insomnia cases and evaluate treatment response. Sleep. 2011; 34:601-608.

[7] King NS, Crawford S, Wenden FJ, Moss NEG, Wade DT. The Rivermead Post Concussion Symptoms Questionnaire: a measure of symptoms commonly experienced after head injury and its reliability. Journal of Neurology. 1995;242:587-592.

[8] Cernovsky ZZ, Mann SC, Velamoor V, Oyewumi LK, Diamond DM, Litman LC. Validation of the Rivermead Post-Concussion Symptoms Questionnaire (RPQ) on Patients Injured in High Impact Car Accidents. Archives of Psychiatry and Behavioral Sciences. 2021;4(1):14-22. doi.org/10.22259/2638-5201.0401003.

[9] Pinfold M, Niere KR, O'Leary EF, Hoving JL, Green S and Buchbinder R. Validity and internal consistency of a Whiplash-Specific disability measure. Spine. 2004;29(3): 263-268.

[10] Blevins CA, Weathers FW, Davis MT, Witte TK, and Domino JL. The Posttraumatic Stress Disorder Checklist for DSM-5 (PCL-5): Development and Initial Psychometric Evaluation. Journal of Traumatic Stress. 2015;28:489-498. doi: 10.1002/jts.22059.

[11] Cernovsky Z, Istasy P, Bureau Y, Chiu S. Scale for retrospective assessment of immediate concussion symptoms. Mental Illness. 2018;10(2):70-71. doi.org/10.4081/mi.2018.7901.
Zack Z. Cernovsky was born in 1947 and holds the Ph.D. in clinical psychology from the University of Zürich, Switzerland, 1986. He is the professor of psychiatry in the medical school of Western University, Canada Professor Cernovsky has published close to 200 scientific articles in the field of psychiatry and medical psychology, and also chapters in university textbooks.

Stephan C. Mann was born on May 6, 1948 in Philadelphia, Pennsylvania, USA. He received his medical degree from the Sidney Kimmel Medical College of Thomas Jefferson University where he also completed his residency in psychiatry. He worked for the United States Veterans Health Administration for over thirty years and retired as Chief of Mental Health \& Behavioral Sciences at the Louisville VA Medical Center, Louisville, KY. He is currently in private practice. Dr. Mann has previously held faculty positions as Professor of Psychiatry at both the University of Pennsylvania School of Medicine and the University of Louisville School of Medicine. He is a Distinguished Life Fellow of the American Psychiatric Association and is certified by the American Board of Psychiatry and Neurology. He has published almost one hundred scientific publications including three books. Dr. Mann's main research focus includes neuroleptic malignant syndrome, malignant catatonia, tardive dyskinesia, and the neurobiology of schizophrenia.

Varadaraj R. Velamoor graduated in Medicine from Osmania University in India and received his training in Psychiatry in the United Kingdom. He is currently Professor of Psychiatry at the Northern Ontario School of Medicine as well as Professor Emeritus at the Western University School of Medicine. He was previously on Faculty at Cornell University. Professor Velamoor has published over a hundred scientific articles as well as book chapters and psychiatric monographs in the areas of suicidal behaviour, violence, stress at the work place, collaborative care, geriatric psychopharmacology and the Neuroleptic Malignant Syndrome (NMS). His work in NMS is internationally recognized and frequently cited. He has received Fellowships from the Royal College of Psychiatrists, UK, as well as the American Psychiatric Association. 